\title{
A NOTE ON THE RANDOM WALK MODEL ARISING IN DOUBLE DIFFUSION
}

\author{
L. H. LIYANAGE, J. M. HILL and C. M. GULATI'
}

(Received 22 June 1981; revised 16 December 1981)

\begin{abstract}
The discrete random walk problem for the unrestricted particle formulated in the double diffusion model given in Hill [2] is solved explicitly. In this model it is assumed that a particle moves along two distinct horizontal paths, say the upper path 1 and lower path 2 . For $:=1,2$, when the particle is in path $i$, it can move at each jump in one of four possible ways, one step to the right with probability $p_{i}$, one step to the left with probability $q_{1}$, remains in the same position with probability $r_{1}$ or exchanges paths but remains in the same horizontal position with probability $s_{1}\left(p_{i}+q_{t}+r_{i}+s_{t}=1\right)$. Using generating functions, the probability distribution of the position of an unrestricted particle is derived. Finally some special cases are discussed to illustrate the general result.
\end{abstract}

\section{Introduction}

The classical random walk model is generalized by Hill [2], so that a particle moves along one of two distinct paths 1 and 2 as follows. For $i=1,2$, the particle is in path $i$ and at each jump it moves one step to the right with probability $p_{i}$, one to the left with probability $q_{i}$, remains in the same position with probability $r_{t}$ or exchanges paths but remains in the same position with probability $s_{i}\left(p_{t}+q_{t}\right.$ $\left.+r_{i}+s_{i}=1\right)$. These probabilities are assumed to be independent of the position of the particle. The purpose of this article is to deduce explicit expressions for the probability distribution of the position of an unrestricted particle. This problem is formulated in Hill [2], but not solved, although means and variances are given. As noted in the review paper Hill [3] the corresponding problems for the two models continuous in time but with discrete space, and continuous in both space and

\footnotetext{
' Department of Mathematics, University of Wollongong, Wollongong, N.S.W. 2500.

(c) Copyright Australian Mathematical Society 1982
} 
time, can however be solved explicitly. Here we give the corresponding expressions for the fully discrete formulation of the model.

Let $u_{k, n}^{i}, v_{k, n}^{i}(i=1,2)$ denote the probabilities that the particle is at position $k$ in paths 1 and 2 respectively at the $n$th step given that its initial position is at the origin in path $i$. For the unrestricted particle we have the following forward equations

$$
\begin{aligned}
& u_{k, n+1}^{i}=p_{1} u_{k-1, n}^{i}+r_{1} u_{k, n}^{i}+q_{1} u_{k+1, n}^{i}+s_{2} v_{k, n}^{i}, \\
& v_{k, n+1}^{i}=p_{2} v_{k-1, n}^{i}+r_{2} v_{k, n}^{i}+q_{2} v_{k+1, n}^{i}+s_{1} u_{k, n}^{i},
\end{aligned}
$$

for $i=1,2$ with the initial conditions

$$
u_{k, 0}^{1}=\delta_{k, 0}, \quad v_{k, 0}^{1}=0, \quad u_{k, 0}^{2}=0, \quad v_{k, 0}^{2}=\delta_{k, 0},
$$

for all integers $k$, for integers $n \geqslant 0$ and where $\delta_{i}$ is the usual Kronecker delta. This formulation differs slightly from the approach of Hill [2]. We solve (1.1) subject to the initial conditions (1.2) in Sections 2 and 3 using generating functions. In the final section, the formulae obtained are illustrated with special cases.

In this section, for comparison we note the solution of the forward equation for the standard random walk model with three possibilities. Given

$$
u_{k, n+1}=p u_{k-1, n}+r u_{k, n}+q u_{k+1, n},
$$

for all integers $k$ and for integers $n \geqslant 0$, the generating function

$$
U_{n}(z)=\sum_{k=-\infty}^{\infty} u_{k, n} z^{k}
$$

satisfies

$$
U_{n+1}(z)=(p z+r+q / z) U_{n}(z)
$$

which gives

$$
U_{n}(z)=\pi(z)^{n},
$$

where $\pi(z)=p z+r+q / z$. Expanding $\pi(z)^{n}$ as a power series of $z$, and taking the coefficient of $z^{k}$, we obtain the probability distribution $u_{k, n}$ given by

$$
u_{k, n}=\sum_{m=m_{0}}^{(n-k) / 2} \frac{n ! p^{m+k} q^{m} r^{n-2 m-k}}{(m+k) ! m !(n-2 m-k) !},
$$

where $m_{0}=\max (0,-k)$ (see Cox and Miller [1], page 26). 


\section{Formulae for generating functions}

Let $U_{n}^{i}(z)$ and $V_{n}^{i}(z)$ denote the $k$-generating functions of the probability distributions $u_{k, n}^{i}$ and $v_{k, n}^{i}$ respectively. For $i=1$ we have from (1.1) and (1.2)

$$
\begin{aligned}
& U_{n+1}^{1}(z)-U_{n}^{1}(z)=\left(\omega_{1}(z)-s_{1}\right) U_{n}^{1}(z)+s_{2} V_{n}^{1}(z), \\
& V_{n+1}^{1}(z)-V_{n}^{1}(z)=\left(\omega_{2}(z)-s_{2}\right) V_{n}^{1}(z)+s_{1} U_{n}^{1}(z),
\end{aligned}
$$

where the functions $\omega_{i}(z)$ are defined by

$$
\omega_{i}(z)=p_{i} z-\left(p_{i}+q_{i}\right)+q_{i} / z \quad(i=1,2)
$$

and the initial conditions are

$$
U_{0}^{1}(z)=1, \quad V_{0}^{1}(z)=0 .
$$

Define the double generating functions

$$
U^{1}(z, \xi)=\sum_{n=0}^{\infty} U_{n}^{1}(z) \xi^{n}, \quad V^{1}(z, \xi)=\sum_{n=0}^{\infty} V_{n}^{1}(z) \xi^{n} .
$$

Then from (2.1) and (2.3) we obtain

$$
\begin{aligned}
\xi U^{\prime}(z, \xi) & =\frac{\left[(1 / \xi-1)-\omega_{2}(z)+s_{2}\right]}{\left\{\left[(1 / \xi-1)-\omega_{1}(z)+s_{1}\right]\left[(1 / \xi-1)-\omega_{2}(z)+s_{2}\right]-s_{1} s_{2}\right\}}, \\
\xi V^{\prime}(z, \xi) & =\frac{s_{1}}{\left\{\left[(1 / \xi-1)-\omega_{1}(z)+s_{1}\right]\left[(1 / \xi-1)-\omega_{2}(z)+s_{2}\right]-s_{1} s_{2}\right\}}
\end{aligned}
$$

Let $\pi_{+}(z)=\left[\pi_{1}(z)+\pi_{2}(z)\right] / 2$ and $\pi_{-}(z)=\left[\pi_{1}(z)-\pi_{2}(z)\right] / 2$ where $\pi_{i}(z)$ is given by

$$
\begin{aligned}
\pi_{i}(z) & =p_{t} z+r_{t}+q_{i} / z \\
& =1+\omega_{i}(z)-s_{i} \quad(i=1,2) .
\end{aligned}
$$

Now

$$
\pi_{ \pm}(z)=P_{ \pm} z+R_{ \pm}+Q_{ \pm} / z
$$

where

$$
P_{ \pm}=\left(p_{1} \pm p_{2}\right) / 2, \quad Q_{ \pm}=\left(q_{1} \pm q_{2}\right) / 2, \quad R_{ \pm}=\left(r_{1} \pm r_{2}\right) / 2,
$$

so that from (2.5) we have

$$
\begin{aligned}
& \xi U^{\prime}(z, \xi)=\frac{\left[1 / \xi-\pi_{+}(z)\right]+\pi_{-}(z)}{\left\{\left[1 / \xi-\pi_{+}(z)\right]^{2}-\left[\pi_{-}(z)^{2}+s_{1} s_{2}\right]\right\}}, \\
& \xi V^{\prime}(z, \xi)=\frac{s_{1}}{\left\{\left[1 / \xi-\pi_{+}(z)\right]^{2}-\left[\pi_{-}(z)^{2}+s_{1} s_{2}\right]\right\}} .
\end{aligned}
$$


On expanding (2.9) as a power series in $\xi$ we have

$$
\begin{aligned}
& U_{n}^{1}(z)=\frac{1}{2}\left\{\left(\theta_{+}^{n}+\theta_{-}^{n}\right)+\frac{\pi_{-}(z)}{\left[\pi_{-}(z)^{2}+s_{1} s_{2}\right]^{1 / 2}}\left(\theta_{+}^{n}-\theta_{-}^{n}\right)\right\}, \\
& V_{n}^{1}(z)=\frac{1}{2}\left\{\frac{s_{1}}{\left[\pi_{-}(z)^{2}+s_{1} s_{2}\right]^{1 / 2}}\left(\theta_{+}^{n}-\theta_{-}^{n}\right)\right\},
\end{aligned}
$$

where

$$
\theta_{+}=\pi_{+}(z)+\left[\pi_{-}(z)^{2}+s_{1} s_{2}\right]^{1 / 2}, \quad \theta_{-}=\pi_{+}(z)-\left[\pi_{-}(z)^{2}+s_{1} s_{2}\right]^{1 / 2} .
$$

The appearance of the square root term in (2.10) makes it difficult to proceed any further in obtaining the probabilities $u_{k, n}^{1}$ and $v_{k, n}^{1}$. In order to overcome this situation we make use of the identities

$$
\theta_{+}^{n} \pm \theta_{-}^{n}=\left[\left(\theta_{+}+\theta_{-}\right) / 2\right]^{n}\left[(1+x)^{n} \pm(1-x)^{n}\right]
$$

where

$$
x=\left(\theta_{+}-\theta_{-}\right) /\left(\theta_{+}+\theta_{-}\right)=\left[\pi_{-}(z)^{2}+s_{1} s_{2}\right]^{1 / 2} / \pi_{+}(z) .
$$

From the above expression we obtain

$$
\frac{\left(\theta_{+}^{n}-\theta_{-}^{n}\right)}{\left[\pi_{-}(z)^{2}+s_{1} s_{2}\right]^{1 / 2}}=2 \pi_{+}(z)^{n} \sum_{l=0}^{L_{0}}\left(\begin{array}{c}
n \\
2 l+1
\end{array}\right) \frac{\left[\pi_{-}(z)^{2}+s_{1} s_{2}\right]^{\prime}}{\pi_{+}(z)^{2 l+1}}
$$

and

$$
\left(\theta_{+}^{n}+\theta_{-}^{n}\right)=2 \pi_{+}(z)^{n} \sum_{l=0}^{L_{1}}\left(\begin{array}{c}
n \\
2 l
\end{array}\right) \frac{\left[\pi_{-}(z)^{2}+s_{1} s_{2}\right]^{l}}{\pi_{+}(z)^{2 l}}
$$

where

$$
\begin{gathered}
L_{0}=n / 2-1, \quad L_{1}=n / 2 \text { for } n \text { even, } \\
L_{0}=L_{1}=(n-1) / 2 \text { for } n \text { odd }
\end{gathered}
$$

These results permit writing $U_{n}^{1}(z), V_{n}^{1}(z)$ as double series in $\pi_{+}(z)$ and $\pi_{-}(z)$. These in turn are expressible as series in $z$ of the form

$$
\begin{aligned}
& \pi_{+}(z)^{n-2 l}=\sum_{f=-(n-2 l)}^{n-2 l} \sum_{i=I_{0}}^{(n-2 l-f) / 2} \frac{(n-2 l) ! P_{+}^{f+i} Q_{+}^{i} R_{+}^{n-2 l-2 i-f_{z} f}}{(f+i) ! i !(n-2 l-2 i-f) !}, \\
& \pi_{-}(z)^{2 m}=\sum_{g=-2 m}^{2 m} \sum_{j=J_{0}}^{(2 m-g) / 2} \frac{2 m ! P_{-}^{g} Q_{-}^{j} R_{-}^{2 m-2 j-g_{z} g}}{(g+j) ! j !(2 m-2 j-g) !},
\end{aligned}
$$


where $P_{ \pm}, Q_{ \pm}$and $R_{ \pm}$are given by (2.8) and

$$
I_{0}=\max (0,-f), \quad J_{0}=\max (0,-g) .
$$

Thus

$$
\pi_{+}(z)^{n-2 l} \pi_{-}(z)^{2 m}=\sum_{k=-(n-2 l+2 m)}^{n-2 l+2 m} \gamma_{k, n-2 l, 2 m} z^{k},
$$

where the coefficients $\gamma_{a, b, c}$ are defined as the convolution

$$
\gamma_{a, b, c}=\sum_{H=H_{0}}^{H_{1}} \alpha_{a-H, b}^{+} \alpha_{H, c}^{-},
$$

where $H_{0}=\max (a-b,-c), H_{1}=\min (a+b, c)$, and

$$
\alpha_{a, b}^{ \pm}=\sum_{k=K_{0}}^{(b-a) / 2} \frac{b ! P_{ \pm}^{k+a} Q_{ \pm}^{k} R_{ \pm}^{b-2 k-a}}{(k+a) ! k !(b-2 k-a) !},
$$

and $K_{0}=\max (0,-a)$. Substituting these results in the series obtained for $U_{n}^{1}(z)$ and $V_{n}^{1}(z)$ and using the double sum

$$
\sum_{j=0}^{N} \sum_{i=0}^{j} a_{\jmath, t}=\sum_{J=0}^{N} \sum_{i=j}^{N} a_{i, t-\jmath},
$$

we obtain

$$
\begin{aligned}
U_{n}^{\prime}(z)= & \sum_{l=0}^{L_{1}} \sum_{m=l}^{L_{1}}\left(\begin{array}{c}
n \\
2 m
\end{array}\right)\left(\begin{array}{c}
m \\
l
\end{array}\right)\left(s_{1} s_{2}\right)^{l} \sum_{k=-(n-2 l)}^{n-2 l} \gamma_{k, n-2 m, 2(m-l)} z^{k} \\
& +\sum_{l=0}^{L_{0}} \sum_{m=l}^{L_{0}}\left(\begin{array}{c}
n \\
2 m+1
\end{array}\right)\left(\begin{array}{c}
m \\
l
\end{array}\right)\left(s_{1} s_{2}\right)^{l} \sum_{k=-(n-2 l)}^{n-2 l} \gamma_{k, n-2 m-1,2(m-l)+1} z^{k} \\
V_{n}^{\prime}(z)= & s_{1} \sum_{l=0}^{L_{0}} \sum_{m=l}^{L_{0}}\left(\begin{array}{c}
n \\
2 m+1
\end{array}\right)\left(\begin{array}{c}
m \\
l
\end{array}\right)\left(s_{1} s_{2}\right)^{l} \sum_{k=-(n-2 l-1)}^{n-2 l-1} \gamma_{k, n-2 m-1,2(m-l)} z^{k}
\end{aligned}
$$

Similar expressions can be obtained for $U_{n}^{2}(z)$ and $V_{n}^{2}(z)$ from $V_{n}^{1}(z)$ and $U_{n}^{1}(z)$ respectively by simply interchanging $p_{1}$ and $p_{2}, q_{1}$ and $q_{2}, r_{1}$ and $r_{2}$ and $s_{1}$ and $s_{2}$.

\section{The probability distribution of the position of the particle}

In this section, the probability distribution of the position of the particle is given. The probabilities $u_{k, n}^{t}$ and $v_{k, n}^{\prime}$ are obtained from the coefficients of $z^{k}$ in 
the generating functions $U_{n}^{\prime}(z)$ and $V_{n}^{i}(z)$ respectively. The final results for $i=1$ are

$$
\begin{aligned}
u_{k, n}^{1}= & \sum_{l=0}^{n / 2-|k / 2|} \sum_{m=l}^{[n / 2]}\left(\begin{array}{c}
n \\
2 m
\end{array}\right)\left(\begin{array}{c}
m \\
l
\end{array}\right)\left(s_{1} s_{2}\right)^{l} \gamma_{k, n-2 m, 2(m-l)} \\
& +\sum_{l=0}^{h} \sum_{m=l}^{[n / 2-1 / 2]}\left(\begin{array}{c}
n \\
2 m+1
\end{array}\right)\left(\begin{array}{c}
m \\
l
\end{array}\right)\left(s_{1} s_{2}\right)^{l} \gamma_{k, n-2 m-1,2(m-l)+1},
\end{aligned}
$$

for $-n \leqslant k \leqslant n$ and where $[y]$ denotes the largest integer less than or equal to $y$ and $h=n / 2-|k / 2|-\delta_{k, 0} / 2$. Further

$$
v_{k, n}^{1}=s_{1} \sum_{l=0}^{h} \sum_{m=l}^{[n / 2-1 / 2]}\left(\begin{array}{c}
n \\
2 m+1
\end{array}\right)\left(\begin{array}{c}
m \\
l
\end{array}\right)\left(s_{1} s_{2}\right)^{l} \gamma_{k, n-2 m-1,2(m-l)},
$$

for $-(n-1) \leqslant k \leqslant(n-1)$. Similarly for $i=2$ we have

$$
u_{k, n}^{2}=s_{2} \sum_{l=0}^{h} \sum_{m=l}^{[n / 2-1 / 2]}\left(\begin{array}{c}
n \\
2 m+1
\end{array}\right)\left(\begin{array}{c}
m \\
l
\end{array}\right)\left(s_{1} s_{2}\right)^{l} \gamma_{k, n-2 m-1,2(m-l)},
$$

for $-(n-1) \leqslant k \leqslant(n-1)$. Further

$$
\begin{aligned}
v_{k, n}^{2}= & \sum_{l=0}^{n / 2-|k / 2|} \sum_{m=l}^{[n / 2]}\left(\begin{array}{c}
n \\
2 m
\end{array}\right)\left(\begin{array}{c}
m \\
l
\end{array}\right)\left(s_{1} s_{2}\right)^{l} \gamma_{k, n-2 m, 2(m-l)} \\
& -\sum_{l=0}^{h} \sum_{m=l}^{[n / 2-1 / 2]}\left(\begin{array}{c}
n \\
2 m+1
\end{array}\right)\left(\begin{array}{c}
m \\
l
\end{array}\right)\left(s_{1} s_{2}\right)^{l} \gamma_{k, n-2 m-1,2(m-l)+1},
\end{aligned}
$$

for $-n \leqslant k \leqslant n$. Thus the probability that the particle is at position $k$, of path 1 at the $n$th step, $u_{k, n}$, is given by

$$
u_{k, n}=u_{0} u_{k, n}^{1}+v_{0} u_{k, n}^{2},
$$

and the probability that the particle is at position $k$ of path 2 at the $n$th step, $v_{k, n}$, is given by

$$
v_{k, n}=u_{0} v_{k, n}^{1}+v_{0} v_{k, n}^{2},
$$

where $u_{0}$ and $v_{0}$ are the probabilities the particle is initially at the origin in paths 1 and 2 respectively.

\section{Special cases and examples}

In this section we illustrate the results obtained with some special cases and examples. Firstly we check the case where $p_{1}=p_{2}=p, q_{1}=q_{2}=q, r_{1}=r_{2}=r$ 
and $s_{1}=s_{2}=s$. This should give a simple random walk model with the probability of the particle being in the same position to be $r+s$. Thus equation (2.8) becomes

$$
P_{+}=p, \quad Q_{+}=q, \quad R_{+}=r, \quad P_{-}=Q_{-}=R_{-}=0,
$$

which gives together with (2.19), (2.20) and (2.21) that

$$
\boldsymbol{\alpha}_{a, b}^{-}=\boldsymbol{\delta}_{a, 0} \boldsymbol{\delta}_{b, 0}, \quad \gamma_{a, b, c}=\alpha_{a, b}^{+} \delta_{c, 0} .
$$

Hence equations (3.1) and (3.2) reduce to, for $-n \leqslant k \leqslant n$,

$$
u_{k, n}^{1}=\sum_{l=0}^{(n-|k|) / 2}\left(\begin{array}{c}
n \\
2 l
\end{array}\right) s^{2 l} \alpha_{k, n-2 l}^{+}
$$

while for $-(n-1) \leqslant k \leqslant(n-1)$

$$
v_{k, n}^{1}=\sum_{l=0}^{h}\left(\begin{array}{c}
n \\
2 l+1
\end{array}\right) s^{2 l+1} \alpha_{k, n-(2 l+1)}^{+} .
$$

Thus the probability that the particle is in position $k$ at the $n$th step, starting from the origin is given by

$$
\begin{aligned}
u_{k, n}^{1}+v_{k, n}^{1} & =u_{k, n}^{2}+v_{k, n}^{2}, \\
& =\sum_{i=0}^{n-|k|}\left(\begin{array}{c}
n \\
i
\end{array}\right) s^{i} \alpha_{k, n-1}^{+}, \\
& =\sum_{m=m_{0}}^{(n-k) / 2} \frac{n ! p^{m+k} q^{m}(r+s)^{n-k-2 m}}{(m+k) ! m !(n-k-2 m) !},
\end{aligned}
$$

where $m_{0}=\max (0,-k)$. Notice that this result does agree with that of the classical random walk model (see (1.7)).

Secondly, consider the case $s_{1}=0$, in which downward transitions are forbidden. Then the equations (3.1) and (3.2) give

$$
u_{k, n}^{1}=\sum_{m=0}^{[n / 2]}\left(\begin{array}{c}
n \\
2 m
\end{array}\right) \gamma_{k, n-2 m, 2 m}+\sum_{m=0}^{[n / 2-1 / 2]}\left(\begin{array}{c}
n \\
2 m+1
\end{array}\right) \gamma_{k, n-(2 m+1), 2 m+1}
$$

and $v_{k, n}^{1}=0$ for all $k$ such that $-n \leqslant k \leqslant n$. Further (4.6) reduces to

$$
u_{k, n}^{1}=\sum_{i=0}^{n}\left(\begin{array}{c}
n \\
i
\end{array}\right) \gamma_{k, n-1, i}
$$

which can be shown to take the form of a simple random walk model with parameters $p_{1}, q_{1}$ and $r_{1}$. Now consider the probability the particle is in position $n$ 
at the $n$th step. From (3.1) and (3.2) we have

$$
\begin{aligned}
u_{n, n}^{1} & =\sum_{m=0}^{[n / 2]}\left(\begin{array}{c}
n \\
2 m
\end{array}\right) \gamma_{n, n-2 m, 2 m}+\sum_{m=0}^{[n / 2-1 / 2]}\left(\begin{array}{c}
n \\
2 m+1
\end{array}\right) \gamma_{n, n-(2 m+1), 2 m+1} \\
& =p_{1}^{n}, \\
v_{n, n}^{1} & =0 .
\end{aligned}
$$

Hence,

$$
u_{n, n}=u_{0} p_{1}^{n}, \quad v_{n, n}=v_{0} p_{2}^{n} .
$$

Similarly we can show that

$$
u_{-n, n}=u_{0} q_{1}^{n}, \quad v_{-n, n}=v_{0} q_{2}^{n} .
$$

Finally we give explicitly the probability distributions for $n=2$ and $n=3$ from (3.1) and (3.2). Using (4.8) we can easily obtain the probabilities $u_{ \pm 2,2}, v_{ \pm 2,2}$, $u_{ \pm 3,3}, v_{ \pm 3,3}$. In order to obtain the remaining probabilities, the necessary values for the $\gamma$ and $\alpha^{ \pm}$functions are given by Tables 1 and 2. For the case $n=2$, which represents an even number of steps, we have after simplification

$$
\begin{aligned}
& u_{0,2}^{1}=r_{1}^{2}+s_{1} s_{2}+2 p_{1} q_{1}, \\
& v_{0,2}^{1}=s_{1} r_{1}+s_{1} r_{2}, \\
& u_{1,2}^{1}=2 p_{1} r_{1}, \quad u_{-1,2}^{1}=2 q_{1} r_{1}, \\
& v_{1,2}^{1}=s_{1} p_{1}+s_{1} p_{2}, \quad v_{-1,2}^{1}=s_{1} q_{1}+s_{1} q_{2} .
\end{aligned}
$$

\section{TABLE 1}

$\gamma_{a, b, c}$ in terms of $\alpha^{ \pm}$functions for special values of $a, b$ and $c$.

\begin{tabular}{|l|l|}
\hline$\gamma_{i, j, 0}$ & $\alpha_{t, j}^{+} \quad(i, j=0,1,2, \ldots)$ \\
$\gamma_{1,0, j}$ & $\alpha_{t, j}^{-} \quad(i, j=0,1,2, \ldots)$ \\
$\gamma_{0,1,1}$ & $\alpha_{1,1}^{+} \alpha_{-1,1}^{-}+\alpha_{0,1}^{+} \alpha_{0,1}^{-}+\alpha_{-1,1}^{+} \alpha_{1,1}^{-}$ \\
$\gamma_{0,1,2}$ & $\alpha_{1,1}^{+} \alpha_{-1,2}^{-}+\alpha_{0,1}^{+} \alpha_{0,2}^{-}+\alpha_{-1,1}^{+} \alpha_{1,2}^{-}$ \\
$\gamma_{0,2,1}$ & $\alpha_{1,2}^{+} \alpha_{-1,1}^{-}+\alpha_{0,2}^{+} \alpha_{0,1}^{-}+\alpha_{-1,2}^{+} \alpha_{1,1}^{-}$ \\
$\gamma_{1,1,1}$ & $\alpha_{1,1}^{+} \alpha_{0,1}^{-}+\alpha_{0,1}^{+} \alpha_{1,1}^{-}$ \\
$\gamma_{1,1,2}$ & $\alpha_{1,1}^{+} \alpha_{0,2}^{-}+\alpha_{0,1}^{+} \alpha_{1,2}^{-}+\alpha_{-1,1}^{+} \alpha_{2,2}^{-}$ \\
$\gamma_{1,2,1}$ & $\alpha_{2,2}^{+} \alpha_{-1,1}^{-}+\alpha_{1,2}^{+} \alpha_{0,1}^{-}+\alpha_{0,2}^{+} \alpha_{1,1}^{-}$ \\
$\gamma_{2,1,2}$ & $\alpha_{1,1}^{+} \alpha_{1,2}^{-}+\alpha_{0,1}^{+} \alpha_{2,2}^{-}$ \\
$\gamma_{2,2,1}$ & $\alpha_{1,2}^{+} \alpha_{1,1}^{-}+\alpha_{2,2}^{+} \alpha_{0,1}^{-}$ \\
\hline
\end{tabular}


TABLE 2

$\alpha^{ \pm}$functions appearing in Table 1 , in terms of $P_{ \pm}, Q_{ \pm}$and $R_{ \pm}\left(\alpha_{0,0}^{ \pm}=1\right)$.

\begin{tabular}{|l|c|c|c|}
\hline$\alpha_{0,1}^{ \pm}$ & $R_{ \pm}$ & $\alpha_{-1,2}^{ \pm}$ & $2 Q_{ \pm} R_{ \pm}$ \\
$\alpha_{1,1}^{ \pm}$ & $P_{ \pm}$ & $\alpha_{2,2}^{ \pm}$ & $P_{ \pm}^{2}$ \\
$\alpha_{-1,1}^{ \pm}$ & $Q_{ \pm}$ & $\alpha_{0,3}^{ \pm}$ & $R_{ \pm}^{3}+6 P_{ \pm}^{ \pm} Q_{ \pm} R_{ \pm}$ \\
$\alpha_{0,2}^{ \pm}$ & $R_{ \pm}^{2}+2 P_{ \pm} Q_{ \pm}$ & $\alpha_{1,3}^{ \pm}$ & $3 P_{ \pm} R_{ \pm}^{2}+3 P_{ \pm}^{2} Q_{ \pm}$ \\
$\alpha_{1,2}^{ \pm}$ & $2 P_{ \pm} R_{ \pm}$ & $\alpha_{2,3}^{ \pm}$ & $3 P_{ \pm}^{2} R_{ \pm}$ \\
\hline
\end{tabular}

For $n=3$, which represents an odd number of steps, simplification yields

$$
\begin{aligned}
& u_{0,3}^{1}=r_{1}^{3}+6 p_{1} q_{1} r_{1}+2 s_{1} s_{2} r_{1}+s_{1} s_{2} r_{2}, \\
& v_{0,3}^{1}=s_{1}\left(r_{1}^{2}+r_{2}^{2}+r_{1} r_{2}+2 p_{1} q_{1}+2 p_{2} q_{2}+p_{2} q_{1}+p_{1} q_{2}\right), \\
& u_{1,3}^{1}=3 p_{1} r_{1}^{2}+3 q_{1} p_{1}^{2}+2 s_{1} s_{2} p_{1}+s_{1} s_{2} p_{2}, \\
& v_{1,3}^{1}=s_{1}\left(2 p_{1} r_{1}+2 p_{2} r_{2}+p_{2} r_{1}+p_{1} r_{2}\right), \\
& u_{-1,3}^{1}=3 q_{1} r_{1}^{2}+3 p_{1} q_{1}^{2}+2 s_{1} s_{2} q_{1}+s_{1} s_{2} q_{2}, \\
& v_{-1,3}^{1}=s_{1}\left(2 q_{1} r_{1}+2 q_{2} r_{2}+q_{2} r_{1}+q_{1} r_{2}\right), \\
& u_{2,3}^{1}=3 r_{1} p_{1}^{2}, \quad v_{2,3}^{1}=s_{1}\left(p_{1}^{2}+p_{1} p_{2}+p_{2}^{2}\right), \\
& u_{-2,3}^{1}=3 r_{1} q_{1}^{2}, \quad v_{-2,3}^{1}=s_{1}\left(q_{1}^{2}+q_{1} q_{2}+q_{2}^{2}\right) .
\end{aligned}
$$

Such expressions might be expected, and provide a check on the general result.

\section{Acknowledgement}

The authors are appreciative of the detailed suggestions of the referee which materially improved the presentation of this paper.

\section{References}

[1] D. R. Cox and H. D. Miller, The theory of stochastic processes (Methuen, London, 1965).

[2] J. M. Hill, "A discrete random walk model for diffusion in media with double diffusivity", $J$. Austral. Math. Soc. Ser. B 22 (1980), 58-74.

[3] J. M. Hill, "A random walk model for diffusion in the presence of high-diffusivity paths", Advances in Molecular Relaxation and Interaction Processes 19 (1981), 261-284. 